\title{
GMPPB-congenital disorders of glycosylation associate with decreased enzymatic activity of GMPPB
}

\author{
Zhe Liu ${ }^{1 \dagger}$, Yan Wang ${ }^{1 \dagger}$, Fan Yang ${ }^{1}$, Qin Yang ${ }^{1}$, Xianming Mo², Ezra Burstein ${ }^{3}$, Da Jia ${ }^{1 *}$, Xiao-tang Cai ${ }^{*}$ and \\ Yingfeng $\mathrm{Tu}^{1 *}$
}

\begin{abstract}
The congenital disorders of glycosylation (CDG) are a family of metabolic diseases in which glycosylation of proteins or lipids is deficient. GDP-mannose pyrophosphorylase B (GMPPB) mutations lead to CDG, characterized by neurological and muscular defects. However, the genotype-phenotype correlation remains elusive, limiting our understanding of the underlying mechanism and development of therapeutic strategy. Here, we report a case of an individual presenting congenital muscular dystrophy with cerebellar involvement, who presents two heterozygous GMPPB mutations (V111G and G214S). The V111G mutation significantly decreases GMPPB's enzymatic activity. By measuring enzymatic activities of 17 reported GMPPB mutants identified in patients diagnosed with GMPPB-CDG, we discover that all tested GMPPB variants exhibit significantly decreased enzymatic activity. Using a zebrafish model, we find that Gmppb is required for neuronal and muscle development, and further demonstrate that enzymatic activity of GMPPB mutants correlates with muscular and neuronal phenotypes in zebrafish. Taken together, our findings discover the importance of GMPPB enzymatic activity for the pathogenesis of GMPPB-CDG, and shed light for the development of additional indicators and therapeutic strategy.
\end{abstract}

Keywords: Congenital disorders of glycosylation, GMPPB, Enzymatic activity, Zebrafish model

\section{Introduction}

The congenital disorders of glycosylation (CDG), a heterogeneous category of disorders, lead to malfunction of multiple organs, especially the nervous system, muscles, and intestines in affected individuals [1-3]. Patients with CDG display a wide spectrum of clinical phenotypes, including but not limited to ataxia, seizures, cerebellar hypoplasia, liver diseases, limb-girdle muscular dystrophies (LGMD), and severe congenital muscular dystrophies (CMD) with eye and brain abnormalities $[1,2,4,5]$. To

\footnotetext{
* Correspondence: Jiada@scu.edu.cn; cxt_1999@126.com; tuyingfeng2haha@sina.com

'Zhe Liu and Yan Wang contributed equally to this work. 'Key Laboratory of Birth Defects and Related Diseases of Women and Children, Department of Paediatrics, State Key Laboratory of Biotherapy, West China Second University Hospital, Sichuan University, Chengdu 610041, China

Full list of author information is available at the end of the article
}

date, mutations in more than 100 genes have been shown to be responsible for CDG [1, 6, 7]. For instance, genetic mutations of phosphomannomutase 2 (PMM2), the enzyme responsible for the conversion of mannose-6phosphate into mannose-1-phosphate, lead to the most common subtype, PMM2-CDG [8, 9]. By far, treatment is not available for most of these disorders. One of the genes that have been recently linked with CDG is guanosine diphosphate mannose (GDP-mannose) pyrophosphorylase B (GMPPB) [10-12]. GMPPB catalyzes the formation of GDP-mannose, using mannose-1-phosphate and GTP as substrates, together with GDP-mannose pyrophosphorylase A (GMPPA). GDP-mannose acts as a sugar donor and participates in four glycosylation pathways, including $\mathrm{N}$ glycosylation, O-mannosylation, C-mannosylation, and glycosylphosphatidylinositol (GPI)-anchor formation [11]. 
To date, more than 50 mutations in GMPPB gene have been identified in affected individuals. Most of the variants are missense, and the remaining mutations include nonsense, frame shift, and splicing. Interestingly, GMPPB mutations-associated phenotypic spectrum ranges from LGMD, to congenital myasthenic syndrome (CMS), to severe CMD with eye and brain symptoms [10-18]. However, there is no clear genotype-phenotype correlation. The mechanism underlying GMPPB mutations-related disorders remains elusive, and no effective therapeutic strategies are available. Given the essential role of GMPPB in catalyzing the formation of GDP-mannose, we hypothesize that the enzymatic activity of GMPPB mutants may correlate with the development of GMPPB-CDG.

In the present study, we report two novel GMPPB mutations (V111G/G214S) in one patient presenting CMD with cerebellar involvement. The V111G mutant displays significantly decreased enzymatic activity. By measuring enzymatic activity of 17 reported GMPPB variants identified in GMPPB-CDG patients, we find that enzymatic activities of these GMPPB variants are significantly impaired, indicating the involvement of compromised activity in the pathogenesis of GMPPB-CDG. This conclusion is further supported by studies using a zebrafish model. Altogether, our study illustrates that impaired GMPPB enzymatic activity might be a consensus causative factor for GMPPB-CDG.

\section{Results}

Identification of novel GMPPB mutations in a patient affected by CMD with cerebellar involvement

The patient was a 1-year old Chinese male, who was diagnosed with CMD with cerebellar involvement (CMD-CRB). His brain showed smaller size of cerebellar hemisphere and enlarged bilateral lateral ventricles, detected by MRI at his fetal stage (34 week), which indicated a possibility of cerebellar hypoplasia (Fig. 1a). Postnatal brain MRI result also revealed an abnormal development of cerebellum (Fig. 1a). The patient also presented with muscle weakness in upper and lower limbs as early as the age of 1 month. He gradually displayed more behavioral problems, including difficulties in raising his head and crawling. Muscle MRI showed edema of gluteus maximus (Fig. 1b). Muscle biopsy indicated chronic myopathy (Fig. 1c). During following-up examination, elevated creatine kinase (CK) was recorded, which fluctuated between 391 and 898 U/l (55-170 U/l) (Table 1), consistent with muscle defects.

Using whole exome sequencing, two compound heterozygous mutations in GMPPB gene (c.332 T > G (p.Val111Gly) and c.640G > A (p.Gly214Ser)) (Fig. 1d) were identified in the patient. The mutations were inherited from his mother and father, respectively, who displayed no obvious abnormality (Fig. 1e). Furthermore, the first fetus of the same parents was also found to have cerebellar atrophy by fetal brain MRI, and the parents chose to abort in accordance with their wills. (Fig. 1e, P2).

\section{CDG-associated GMPPB variants exhibit significantly decreased enzymatic activity}

To test whether enzymatic activity of GMPPB variants could be a determinant for the development of GMPPBCDG, we developed an assay to measure enzymatic activity of GMPPB. GMPPB catalyzes the conversion of GTP and mannose-1-p to GDP-mannose and pyrophosphoric acid. Q-ion exchange chromatography was used to separate GDP-mannose and GTP, due to their difference in negative charge, and the amount of GDPmannose was used to assess activity of GMPPB. Consistent with essential roles of $\mathrm{Mg}^{2+}$ or mannose-1-p in the reaction [11, 19], GDP-mannose couldn't be produced in the absence of $\mathrm{Mg}^{2+}$ or mannose-1-p (Fig. 2a). As shown in Fig. 2b, the amount of GDP-mannose produced was nearly linear with time for the initial $5 \mathrm{~min}$, and the reaction reached saturation within $20 \mathrm{~min}$. Furthermore, GMPPB catalyzed the reaction in a dose-dependent manner (Fig. 2b). These results indicated that our assay is effective to evaluate GMPPB activity. We then sought to determine the effects of GMPPB mutations identified in our patient on its activity. Interestingly, the V111G mutant decreased enzymatic activity by $\sim 60 \%$, while the activity of G214S was comparable to that of GMPPB wild-type (WT) (Fig. 2c). We also examined the effects of the missense mutations on the subcellular localization of GMPPB. We found that V111G mutation caused increased nuclear localization of GMPPB, while G214S mutant seemed to lose nuclear localization (Supplementary Fig. 1).

Next, we selected 17 reported GMPPB missense mutants identified in patients, who displayed symptoms ranged from mild to severe conditions. These GMPPB variants were expressed in E.coli, and their enzymatic activity was measured. All the tested mutants displayed markedly decreased enzymatic activities, with P32L and V254M being the lowest ( 10\% of GMPPB WT) (Fig. 2d). These results indicated that impaired enzymatic activity of GMPPB might be a consensus causative factor for GMPPB-CDG.

\section{Gmppb is required for neuronal and muscle development in zebrafish}

To determine the functions of GMPPB in development, we chose zebrafish as a model organism. Semiquantitative RT-PCR indicated that gmppb could be detected at as early as the one-cell stage. Expression of gmppb gradually increased at bud stage, and peaked at $48 \mathrm{~h}$ post fertilization (hpf), suggesting a role in early embryonic development. (Fig. 3a). Whole mount in situ 
a

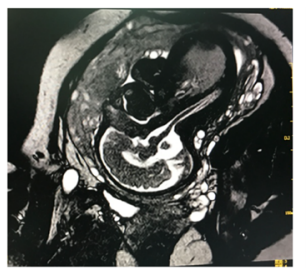

1 Month

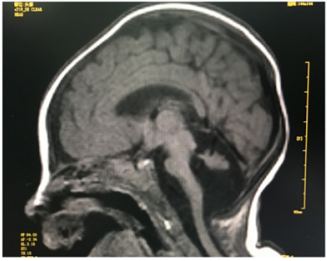

b

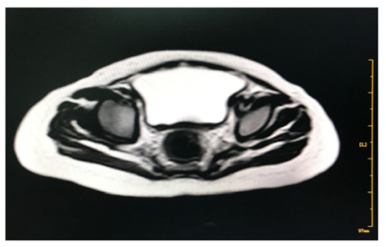

d

GMPPB c.640G $>A$ GT TACAGGGTGAGGC
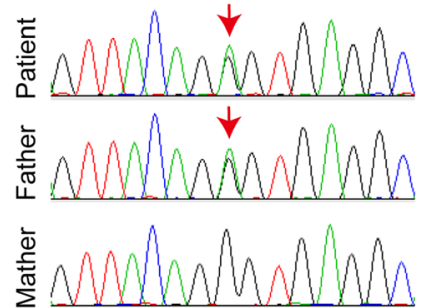
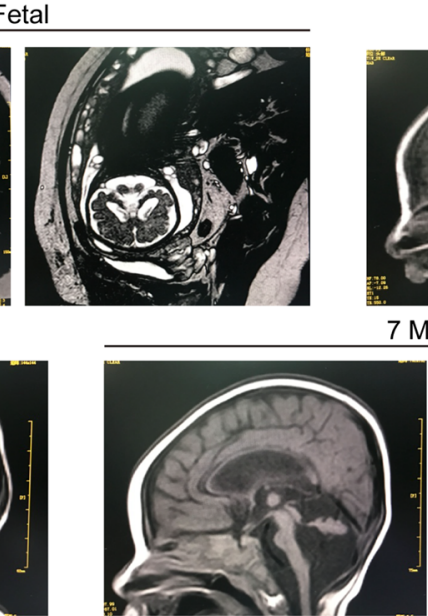

c

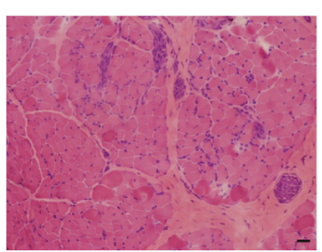

GMPPB c. $332 T>G$ A G T G AC GT G A T C T GC

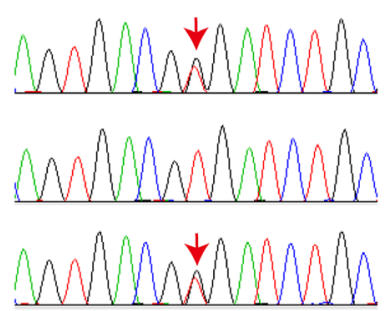

Birth

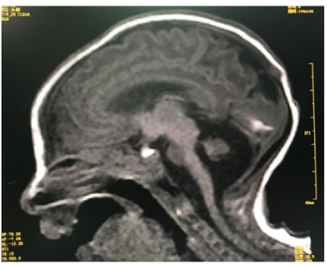

Months
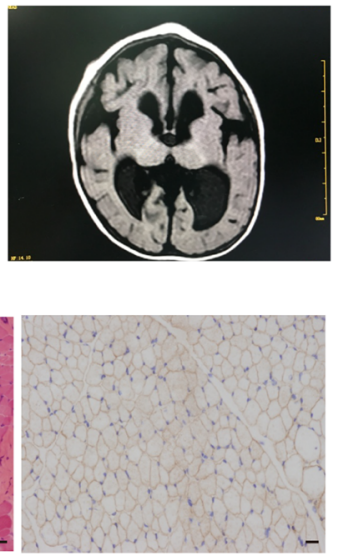

e

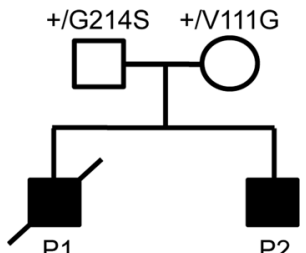

G214S/V111G

Fig. 1 Clinical and genetic characterization. a Brain MRI of the patient at fetal 34 weeks, birth, 1 month, and 7 months, revealing evidence of cerebellar hypoplasia. b Muscle MRI in the right gluteus maximus muscle at 14 months. c Muscle biopsy from gastrocnemius at 14 months. The diameter of muscle fibers range between 5 and $50 \mu \mathrm{m}$, with occasional degenerated and regenerated muscle fibers. Scale bar: $50 \mu \mathrm{m} \mathbf{d}$ Sequence chromatograms of GMPPB gene of the patient. Variations and corresponding WT sequences are shown. The patient carries two missense mutations of GMPPB gene (arrows), inherited from his mother (c.332T > G) and father (c.640G > A), respectively. e Pedigrees

hybridization (WISH) showed that spatial expression of gmppb was ubiquitous during embryonic development (Fig. 3b). Using gmppb-specific morpholino (MO), we were able to successfully deplete most Gmppb protein in gmppb-MO-injected embryos (Fig. 3c). Consistent with the previous study [13], we also observed sparse and disordered muscle fibers in gmppb-MO-injected embryos (Fig. 3d and Supplementary Fig. 2). Furthermore, immunofluorescence assay revealed that the level of $\mathrm{HuC}$ (elavl3), an early marker of pan-neuronal cells, was remarkably decreased in the MO embryos. Importantly, the decrease of $\mathrm{HuC}$ could be effectively rescued by coinjection of mRNA encoding human GMPPB WT (Fig. 3e). Analogous to patients with GMPPB-CDG, gmppb MO-injected zebrafish exhibited remarkably decreased motor ability, as shown by reduced moving distance and moving speed (Fig. 3f).

Given muscle defects and movement disability of patients with GMPPB-CDG and of gmppb MO-injected embryos, we suspected that Gmppb might be involved in development of motor neurons. Thus, we chose $\mathrm{Tg}$ [hb9: GFP] ${ }^{\mathrm{m} / 2}$ transgenic zebrafish to examine morphology of CaP motor neurons [20, 21]. These neurons project ventrally in the middle of each spinal cord, and can be readily examined. Injection of gmppb $\mathrm{MO}$ led to shorter $\mathrm{CaP}$ axons at 24 and $48 \mathrm{hpf}$, which were less 


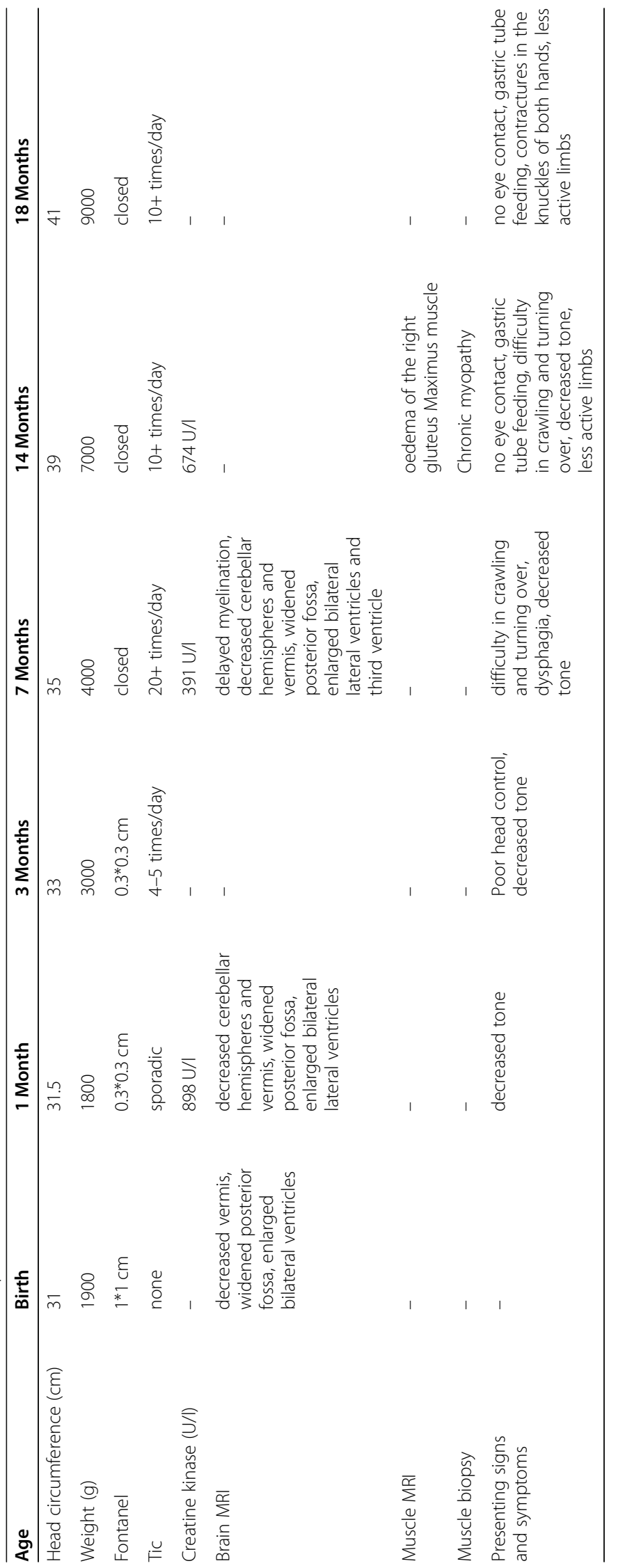


a

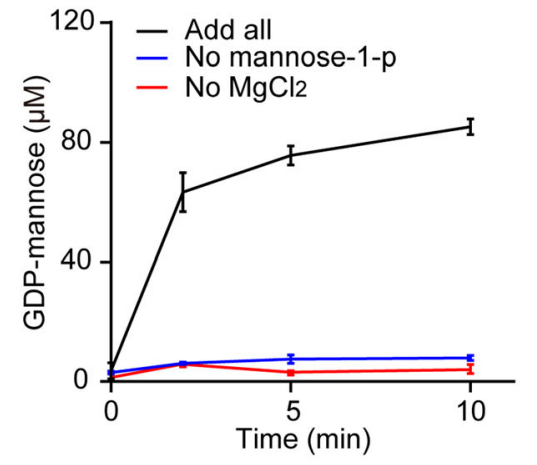

b

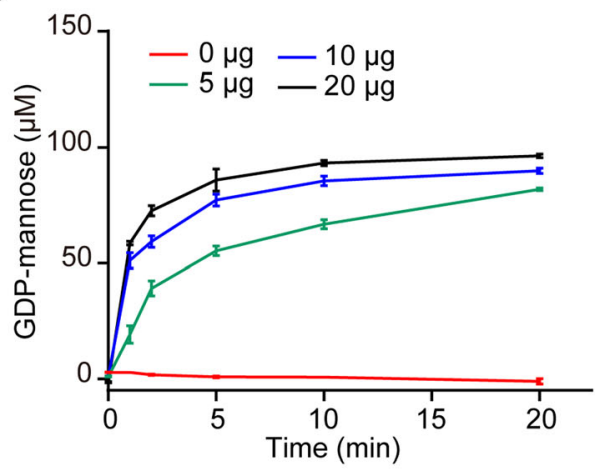

C

d
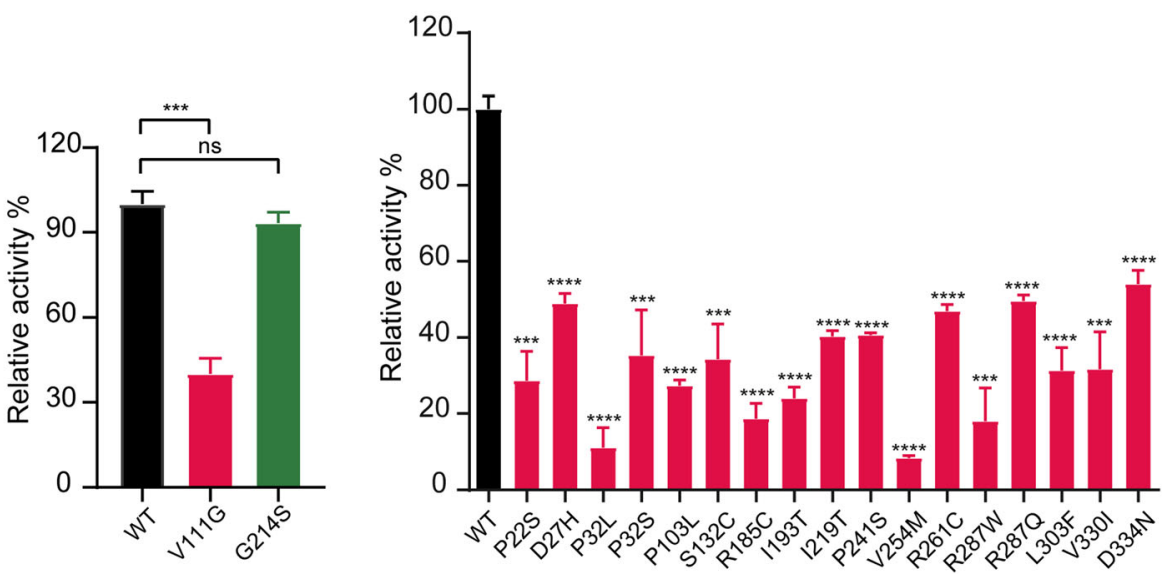

Fig. 2 GMPPB mutants exhibit significantly decreased enzymatic activity. a-b Validation of the designed system for evaluating GMPPB's enzymatic activity. Absence of $\mathrm{Mg}^{2+}$ or substrate mannose-1-p leads to no detectable formation of GDP-mannose (a). The time course of GDP-man production catalyzed by variant doses of GMPPB (b). $0 \mu \mathrm{g}, 5 \mu \mathrm{g}, 10 \mu \mathrm{g}$ or $20 \mu \mathrm{g}$ purified GMPPB was used to catalyze the reaction, which was terminated at the indicated time. The reaction mixtures were subjected to Q-ion exchange chromatography, and the amount of GDP-mannose was determined. c V111G mutation causes significantly decreased activity of GMPPB. Purified GMPPB WT, GMPPB V111G, or GMPPB G214S were subjected to activity detection. Compared to the WT, V111G mutation caused significant reduction of GMPPB activity, whereas GMPPB G214 variant retained activity comparable to WT. $\mathbf{d}$ Disease-associated GMPPB mutation results in significantly decreased enzymatic activity. 17 reported GMPPB mutants identified in patients were expressed in E.coli and subjected to activity detection. All the tested mutants exhibited significantly compromised activity relative to GMPPB WT. Mean \pm SD, ${ }^{* * * *} p<0.0001 ;{ }^{* * *} p<0.001 ;{ }^{* *} p<0.01 ;{ }^{*} p<0.05$. $p$ values were calculated using one-way ANOVA, Tukey's multiple comparisons test

than half of WT. Again, the axonal phenotype could be rescued to the control level by co-injection of mRNA encoding human GMPPB WT (Fig. 3g). Taken together, Gmppb is essential for neuronal and muscle development in zebra fish.

\section{Enzymatic activity of GMPPB variants correlates with muscular and neuronal phenotypes in zebrafish}

Finally, we sought to determine whether enzymatic activity of GMPPB variant correlates with phenotypes in zebrafish. gmppb MO was co-injected with mRNA encoding human GMPPB WT, V111G, or G214S. G214S, which displayed enzymatic activity similar to GMPPB WT, could rescue axonal phenotypes at 24 and $48 \mathrm{hpf}$.
In contrast, V111G, which exhibited significantly decreased activity, failed to do so (Fig. 4a and b). Furthermore, GMPPB WT and G214S, but not V111G, were able to restore the muscular phenotypes of MO-injected embryos, which displayed sparse and disordered muscle fibers (Fig. 4c, d and Supplementary Fig. 3). Altogether, these data indicated that enzymatic activity of GMPPB is critical for muscular and neuronal development.

To corroborate our conclusion, we chose two additional GMPPB mutations, R261C and P32L, which displayed $50 \%$ and $10 \%$ enzymatic activity of WT, respectively. Coinjection of R261C partially restored decreased length of $\mathrm{CaP}$ neurons caused by GMPPB MO; in contrast, GMPPB P32L was unable to rescue (Fig. 4e). Thus, enzymatic 


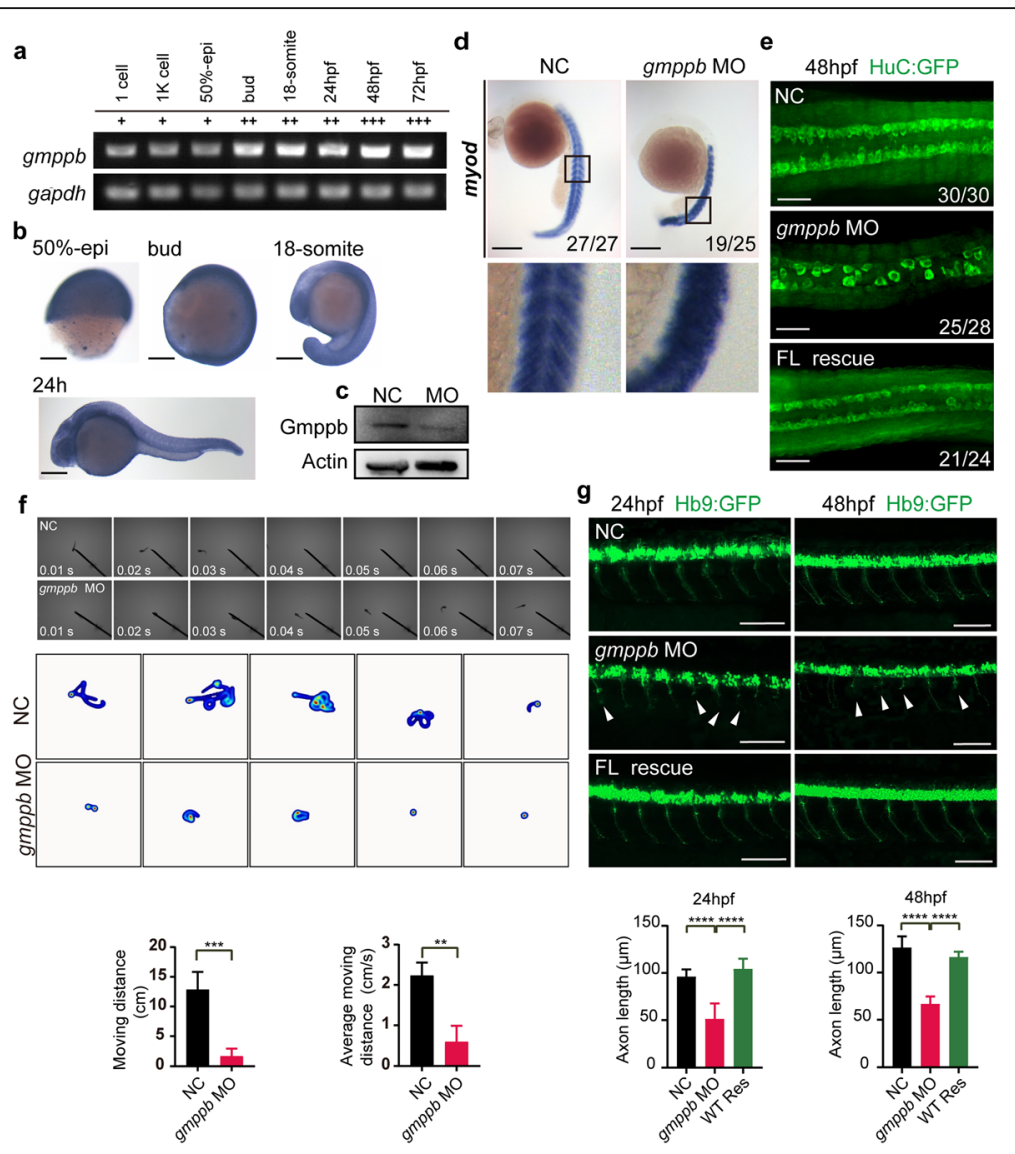

Fig. $3 \mathrm{Gmppb}$ knockdown(KD) in Zebrafish causes abnormal development of muscle and motor neurons, movement disability. a Temporal expression of zebrafish gmppb from fertilization to day 3, and gapdh was used as control. b WISH shows the spatial expression pattern of gmppb using a dig-labeled antisense probe. gmppb was ubiquitously expressed in zebrafish embryos from 50\%-epi to 24hpf. Scale bar: $250 \mu \mathrm{m}$. c Immunoblot of entire zebrafish tissue extracts indicates that gmppb MO injection effectively decreased Gmppb protein level. MO injection was performed at the one cell stage. Actin was used as a loading control. d Expression of myod in zebra fish, with or without gmppb MO injection, determined by WISH. The black rectangles label the position of enlarged views (bottom). Scale bar: $250 \mu \mathrm{m}$. e Gmppb KD results in decreased HuC (green) expression. Tg [HuC: GFP] transgenic zebrafish was injected with control MO (NC), or gmppb MO alone or together with mRNA encoding GMPPB. HuC expression was observed at 48 hpf. Scale bar: $100 \mu \mathrm{mf}$ gmppb mutant larvae display decreased motor ability. Representative images and summarized movement path of zebrafish injected with control MO (NC) or gmppb MO. (Upper). Bar graph summarizes the moving distance and moving speed of zebrafish injected with control MO (NC) or gmppb MO (Lower). Motor ability was monitored in 2 dpf larvae. g. GMPPB is required for motor neuronal development in zebra fish. Tg [hb9: GFP] ${ }^{\mathrm{ml} 2}$ transgenic zebrafish was injected with control $\mathrm{MO}(\mathrm{NC})$, or gmppb MO alone or together with mRNA encoding GMPPB at one-cell stage. Top: morphology of CaP axons was observed at 24 and 48hpf; bottom: statistical results of the length of CaP axons. Scale bar: $100 \mu \mathrm{m}$. Mean $\pm \mathrm{SD},{ }^{* * * *} p<0.0001 ;{ }^{* * *} p<0.001 ;{ }^{* *} p<$ $0.01 ;{ }^{*} p<0.05$. $p$ values were calculated using one-way ANOVA, Tukey's multiple comparisons test

activity of GMPPB might be a critical determinant for phenotypes of zebrafish.

\section{Discussion}

So far, more than 50 different GMPPB mutations have been reported. The clinical spectrum of GMPPB-CDG spans from CMS, LGMD to CMD with or without brain abnormalities [10, 13, 14, 18, 22]. However, the mechanism underlying this broad phenotypic spectrum remains unknown. In the present study, we report two novel GMPPB mutations in a patient diagnosed with CMD. We identify enzymatic activity of GMPPB as a key determinant of GMPPB-CDG development. And we find that enzymatic activity of GMPPB variant correlates its ability in rescuing muscular and neuronal phenotypes in zebrafish. Our study suggests that the cellular level of GDP-mannose could correlate with patients' symptoms.

To evaluate the effects of GMPPB mutations on its enzymatic activity, WT GMPPB and its mutants were expressed and further purified as MBP-fusion protein. MBP tag $(\sim 42 \mathrm{kDa})$ is a relatively big tag and might affect protein function [23]. We tried to remove the MBP tag of the fusion protein, which unfortunately produced insoluble protein. We also expressed GMPPB 
with a His6-tag, but did not obtain soluble protein. As the MBP-tagged GMPPB catalyzes the formation of GDP-mannose in time and concentration dependent manners, it is the best available approach to compare the activity of wild-type and mutants proteins.

GMPPB is comprised of two functional domains: Nterminal catalytic domain (residues 1-250) and a lefthanded parallel beta-helix domain (residues 251-360) [18]. Whereas disease-causing mutations are found through the entire GMPPB protein, mutations within its catalytic domain impair activity more severely than those in the $\mathrm{LbH}$ domain. Consequently, individuals carrying these variants display more severe symptoms. How do GMPPB mutations affect its functions? Previous studies suggested that some mutants, such as D334N, P22S, P32L, R287Q, V330I, V254M, R287W, I268T, and G354S, altered subcellular localization of GMPPB [10, 13, 18]. And V111G and G214S mutations identified in our patient also altered subcellular localization of GMPPB (Supplementary Fig. 1). Furthermore, some mutations, including V254M,. R287W, I268T, and G354S, impaired GMPPB protein level [10, 18]. Interestingly, reduced protein levels of I268T and G354S could be rescued by lysosomal inhibitors, indicating the involvement of autophagy-lysosomal pathway in destabilization of GMPPB [18]. The disease-causing mechanisms of other mutations warrant further investigation.

Significant efforts have been spent in the past in order to understand the underlying mechanism of each subtype of CDG and to develop specific treatment strategy. For instance, prenatal supplementation of mannose successfully overcomes embryonic lethality of Pmm2 mice, and has been proposed to treat PMM2-CDG [24, 25]. However, mannose supplementation failed to rescue lethality in a mice bearing the R137H/F115L genotype (corresponding to the two most prevalent mutations in patients with PMM2CDG: R141H and F119L). Both PMM2-CDG mouse model and PMM2-CDG patient-derived fibroblasts were reported to display decreased PMM activity, GDP-mannose and total cellular protein glycosylation [26]. And supplementation of mannose, the most upstream substrate, might not be sufficient to compensate the severely impaired PMM2 activity, contributing to its inability to rescue lethality in PMM2 R137H/F115L mice. During our efforts to search for therapeutic regimen for GMPPB-CDG, we found that supplementation of GDP-mannose effectively rescues the phenotypes in zebrafish model (unpublished data in our complementary paper). However, glucose or mannose, the most upstream substrate, failed to rescue muscle defects and abnormal development of motor neurons observed in gmppb MO-injected embryos. Our study demonstrates GMPPB's enzymatic activity as a potential causative factor for GMPPB-CDG, and suggests that supplementation of GDP-mannose or its analogs may represent a potential therapeutic strategy for GMPPB-CDG.

\section{Materials and methods Genetic analysis}

Genomic DNA was extracted from blood using standard procedures. Whole exome sequencing was performed for the patient as previously described [27]. The obtained sequences were mapped to human genome build hg19.Variants were called using LifeScopeTM 2.5 (Life Technologies). Variants were then filtered using ANNOVAR [28] against the ENCODE GencodeV14 [29].

\section{Muscle pathology}

We performed muscle biopsy on the right gastrocnemius in the patient at 14 months. The muscle tissue was frozen and cut at $7 \mu \mathrm{m}$. Sections were stained with Hematoxylin and Eosin (HE). Sections were also immunostained with the antibody against dystrophin with standard procedures [18].

\section{Protein expression and purification}

Human GMPPB cDNA was cloned into a modified pMAL vector with an $\mathrm{N}$-terminal MBP tag. GMPPB mutants were generated by site-directed mutagenesis. Expression of GMPPB WT or mutants was induced in BL21 (DE3) cells by $0.5 \mathrm{mM}$ isopropyl $\beta$-D-1-thiogalactopyranoside (IPTG) at $16^{\circ} \mathrm{C}$ for $12 \mathrm{~h}$. Cells were harvested, and re-suspended with the lysis buffer $(20 \mathrm{mM}$ Tris- $\mathrm{HCl}, \mathrm{pH} 8.0,200 \mathrm{mM}$ $\mathrm{NaCl}, 1 \mathrm{mM}$ DTT, 1\% PMSF). Cells were broken by highpressure homogenizer, and subjected to centrifugation. Proteins were purified with amylose-affinity resin, and eluted into Buffer 1 (20 mM Tris- $\mathrm{HCl}, \mathrm{pH}$ 8.0, $200 \mathrm{mM} \mathrm{NaCl}, 1$ $\mathrm{mM}$ DTT) supplemented with $1 \%(\mathrm{w} / \mathrm{v})$ maltose.

\section{Enzymatic activity assay}

For enzymatic activity assays, purified GMPPB protein $(10 \mu \mathrm{g})$ was mixed with $0.5 \mathrm{mM}$ Man-1-P, $0.25 \mathrm{mM}$ GTP, $50 \mathrm{mM}$ Tris, $\mathrm{pH} 7.5$, and $1 \mathrm{mM} \mathrm{MgCl}$. The reaction was carried out at $37^{\circ} \mathrm{C}$ for $5 \mathrm{~min}$, and terminated by the addition of $\mathrm{NaOH}(20 \mu \mathrm{l}, 10 \mathrm{M})$. After termination, the reaction mixtures were subjected to Q-ion exchange chromatography, which separated GDP-mannose from GTP. The eluted positions of GDP-mannose and GTP were also confirmed using pure chemicals. The amount of GDPmannose and GTP were determined by integrating their absorption at $260 \mathrm{~nm}$. All experiments were conducted at least three times.

\section{Immunofluorescence}

C2C12 transfected with indicated constructs were fixed in $4 \%$ paraformaldehyde for $15 \mathrm{~min}$ at room temperature, and then permeabilized with $0.25 \%$ Triton X-100 for $15 \mathrm{~min}$. The permeabilized cells were blocked with 5\% BSA for $1 \mathrm{~h}$ at room temperature and incubated with primary antibodies against HA (proteintech, $66,006-2-I g)$ at $4{ }^{\circ} \mathrm{C}$ overnight. Cells were then incubated 


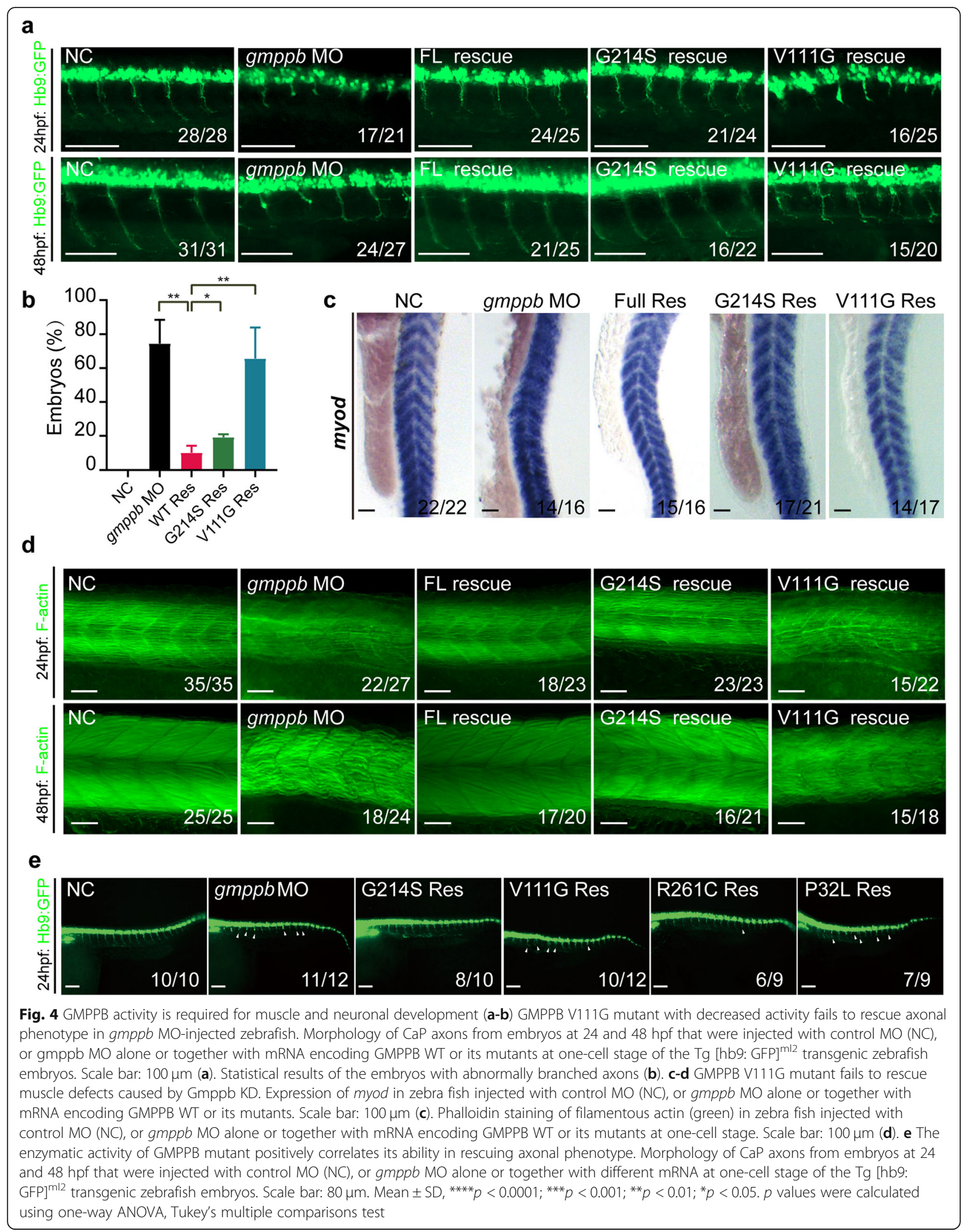


with Alexa Fluor 546-labeled goat-anti-mouse secondary antibody for $1 \mathrm{~h}$. The cells were further counterstained with DAPI to visualize nucleus. Images were taken with equal exposure time.

\section{Zebrafish maintenance}

All zebrafish experiments were performed according to standard procedures. Adult fish and embryos were raised at $27.5-28.5^{\circ} \mathrm{C}$ in the Aquatic Ecosystems. The lines used in this study include: $A B$ strain (wild-type), $\operatorname{Tg}[\mathrm{hb} 9$ : GFP $]^{\mathrm{ml} 2}$ transgenic lines and $\mathrm{Tg}[\mathrm{HuC}: \mathrm{GFP}]$ strain. All experimental protocols were proved by the Animal Ethics Committee, West China Second University Hospital.

\section{Antisense RNA probes and mRNAs}

Antisense RNA probes for in situ hybridization were synthesized in vitro using the Riboprobe system kit (Promega). DNA sequences encoding human GMPPB WT or mutants were cloned into a pcDNA3.1+ vector, and were used as the template for Capped mRNAs synthesis using T7 mMESSAGE mMACHINE kit (Ambion).

\section{Zebrafish knockdown and mRNA injection}

To knock down zebrafish gmppb, we chose a morpholino oligonucleotide (MO) (sequence: 5'-GGACCAGC TGAAAACAGAAACAGAT-3'), which was reported to efficiently deplete Gmppb [13]. Control MO is a standard mismatched control. MO and mRNAs were injected into the yolk and the cell at the stage of one cell, with 5 ng of $\mathrm{MO}$ and/or $200 \mathrm{pg}$ of mRNA per injection.

\section{Total RNA isolation and semi-quantitative RT-PCR}

RNA isolation and Semi-quantitative RT-PCR was carried out as previously described [30]. Fifty zebrafish embryos at indicated time point were collected for each sample. The embryos were grounded, and tissue debris was removed. Total RNA was isolated by RNeasy Plant Mini Kit (FOREGRNE), and then used to synthesize cDNA with the Prime Script Reverse-transcription PCR kit (TaKaRa DRR014A). The resultant cDNA was used as a template for RT-PCR, performed with the Real Master Mix Kit (Roche).

\section{Protein extraction and Western blot}

Zebrafish embryos injected with control or gmppb MO were collected at the stage of $24 \mathrm{~h}$ and subjected to protein extraction. Briefly, after removal of oolemma and yolk, the embryos were lysed with RIPA buffer supplemented with $1 \%$ protease inhibitor mixture (Bimake, B14001) for $30 \mathrm{~min}$ and sonicated at $4{ }^{\circ} \mathrm{C}$. After incubating on ice for $15 \mathrm{~min}$, the lysate were centrifuged at $10000 \mathrm{rpm}$ for $30 \mathrm{~min}$ at $4{ }^{\circ} \mathrm{C}$. The supernatant were collected and protein concentration was measured by the bicinchoninic acid method. The supernatant were boiled in loading buffer and subjected to western blot analysis with the indicated antibodies (GMPPB, proteintech 15,094-1-AP; actin, Abclonal, AC026).

\section{Motor ability}

Larval motor ability was measured on the second day of development (5 larva per group). Single larva was placed in a $10-\mathrm{cm}$ dish, stimulated at the head by a tip of syringe, and its movement path was recorded. Data were analyzed using EthoVision software (Noldus, Inc.).

\section{Whole-mount in situ hybridization (WISH)}

WISH analysis was performed as previously described [31]. In brief, samples at $24 \mathrm{hpf}$ and $48 \mathrm{hpf}$ were dechorionated, fixed with $4 \%$ paraformaldehyde (PFA), dehydrated in methanol (30\% to $100 \%)$ overnight, and rehydrated with methanol (100\% to 30\%). Zebra fish embryos were incubated with proteinase $\mathrm{K}(1 \mathrm{mg} / \mathrm{mL}$, Promega) for 20 $\mathrm{min}$ at room temperature, and then incubated with digoxigenin (DIG)-labeled antisense RNAs (gmppb, myod) at $65^{\circ} \mathrm{C}$ overnight. After the probes were removed, the samples were incubated with alkaline phosphatase (AP)-conjugated anti digoxigenin antibody (Roche, 11,093,274,910, 1: 2000) at $4{ }^{\circ} \mathrm{C}$ overnight. After washing, the samples were subjected to NBT/BCIP (Roche) staining according to the manufacturer's instructions.

\section{Whole-mount immunofluorescence}

Immunofluorescence was performed as previously described [20]. Briefly, zebrafish embryos were fixed with $4 \%$ PFA at $4{ }^{\circ} \mathrm{C}$ overnight, washed 10 times with PBS for 5 min each wash, incubated with acetone at $-20^{\circ} \mathrm{C}$ for $10 \mathrm{~min}$, and finally washed 10 times with PBS for $5 \mathrm{~min}$ each wash. Samples were blocked with 5\% BSA containing $0.1 \%$ triton $\mathrm{X}-100$, and incubated with phalloidin (Sigma, P5282, 1;2000) or Huc (Abcam, ab210554, 1: 250) at $4{ }^{\circ} \mathrm{C}$ overnight. The embryos were then washed, and DAPI was used for nucleic acid staining. The embryos were examined by a Zeiss confocal laser-scanning microscope.

\section{Statistical analysis}

All zebrafish and biochemical experiments were performed at least three times. Statistical analyses were performed using one-way ANOVA, Tukey's multiple comparisons test incorporated in Prism 7 (GraphPad Software). ${ }^{* * *} P<0.001,{ }^{* *} P<0.01,{ }^{*} P<0.05$, ns: not significant.

\section{Supplementary Information}

The online version contains supplementary material available at https://doi. org/10.1186/s43556-021-00027-2.

Additional file 1: Figure S1. V111G and G214S mutations alter subcellular localization of GMPPB. C2C12 myoblasts were transfected with 
HA-GMPPB WT, HA-GMPPB V111G, HA-GMPPB G214S or HA-GMPPB D334N. Compared to WT GMPPB, V111G mutant exhibited increased localization in the nucleus, while G214S mutant seemed to lose nuclear localization. D334N, previously reported to form aggregates within the cytoplasm, was used as a control. Scale bar: $10 \mu \mathrm{m}$. Figure S2. GMPPB $\mathrm{KD}$ causes muscle defects in zebra fish. (a) Phalloidin staining (red) of filamentous actin in zebra fish injected with control MO (NC), or gmppb MO at one-cell stage. Scale bar: $250 \mu \mathrm{m}$ (b) Phalloidin staining (green) on the cross-sections of 24 hpf embryos injected with control MO (NC), or gmppb MO at one-cell stage. Scale bar: $100 \mu \mathrm{m}$. Figure S3. GMPPB V111G mutant fails to rescue muscle defects in zebra fish caused by GMPPB KD. Phalloidin (green) and DAPI (blue) staining on the crosssections of 48 hpf embryos injected with control MO (NC), or gmppb MO alone or together with mRNA encoding GMPPB WT or its mutants at one-cell stage. Scale bar: $100 \mu \mathrm{m}$

\section{Abbreviations}

GDP-mannose: Guanosine diphosphate mannose; GMPPB: GDP-mannose pyrophosphorylase B; CDG: Congenital disorders of glycosylation; LGMD: Limb-girdle muscular dystrophies; CMD: Congenital muscular dystrophies; PMM2: Phosphomannomutase 2; GMPPA: GDP-mannose pyrophosphorylase A; GPI: Glycosylphosphatidylinositol; CMS: Congenital myasthenic syndrome; CMD-CRB: CMD with cerebellar involvement; CK: Creatine kinase; WISH: Whole mount in situ hybridization; MO: Morpholino; HE: Hematoxylin and Eosin

\section{Acknowledgements}

We thank members of our laboratory for helpful discussions.

\section{Code availability}

Not applicable.

\section{Authors' contributions}

Z.L. carried out zebrafish studies with assistance from Q.Y., Y.W. performed biochemical work, and X.C. diagnosed the patient. X.M. provided technical assistance. Y.T. and D.J. supervised the project and prepared the manuscript. The author(s) read and approved the final manuscript.

\section{Funding}

This research is supported by Natural Science Foundation of China (NSFC) grants (\#91854121, \#31871429, \#81901281), National Key Research and Development Program of China (2018YFC1005004), and Sichuan Science and Technology Program (2018RZ0128).

\section{Availability of data and materials}

All data generated or analyzed during this study are included in this published article and its supplementary information files.

\section{Ethics approval and consent to participate}

The study is approved by the ethics committee of West China Second University Hospital, Sichuan University.

All participants provided written informed consent.

\section{Consent for publication}

All participants provided written informed consent

\section{Competing interests}

The authors declare no competing interests.

\section{Author details}

${ }^{1}$ Key Laboratory of Birth Defects and Related Diseases of Women and Children, Department of Paediatrics, State Key Laboratory of Biotherapy, West China Second University Hospital, Sichuan University, Chengdu 610041, China. ${ }^{2}$ Department of Pediatric Surgery and Laboratory of Stem Cell Biology, State Key Laboratory of Biotherapy, West China Hospital, Sichuan University, Chengdu 610041, China. ${ }^{3}$ Department of Internal Medicine, University of Texas Southwestern Medical Center, Dallas, TX 75390, USA.
Received: 30 October 2020 Accepted: 12 January 2021

Published online: 10 May 2021

\section{References}

1. Chang IJ, He M, Lam CT. Congenital disorders of glycosylation. Ann Transl Med. 2018;6(24):477. https://doi.org/10.21037/atm.2018.10.45.

2. Reily C, Stewart TJ, Renfrow MB, Novak J. Glycosylation in health and disease. Nat Rev Nephrol. 2019;15(6):346-66. https://doi.org/10.1038/s41581019-0129-4.

3. de Lonlay P, Seta N, Barrot S, Chabrol B, Drouin V, Gabriel BM, et al. A broad spectrum of clinical presentations in congenital disorders of glycosylation I: a series of 26 cases. J Med Genet. 2001;38(1):14-9. https://doi.org/10.1136/ jmg.38.1.14.

4. Godfrey C, Foley AR, Clement E, Muntoni F. Dystroglycanopathies: coming into focus. Curr Opin Genet Dev. 2011;21(3):278-85. https://doi.org/10.1016/ j.gde.2011.02.001.

5. Taniguchi-Ikeda M, Morioka I, lijima K, Toda T. Mechanistic aspects of the formation of alpha-dystroglycan and therapeutic research for the treatment of alpha-dystroglycanopathy: a review. Mol Asp Med. 2016;51:115-24. https://doi.org/10.1016/j.mam.2016.07.003.

6. Jaeken J, Péanne R. What is new in CDG? J Inherit Metab Dis. 2017:40(4): 569-86. https://doi.org/10.1007/s10545-017-0050-6.

7. Freeze HH, Chong JX, Bamshad MJ, Ng BG. Solving glycosylation disorders: fundamental approaches reveal complicated pathways. Am J Hum Genet 2014;94(2):161-75. https://doi.org/10.1016/j.ajhg.2013.10.024.

8. Vals MA, Pajusalu S, Kals M, Magi R, Ounap K. The prevalence of PMM2-CDG in Estonia based on population carrier frequencies and diagnosed patients. JIMD Rep. 2018:39:13-7. https://doi.org/10.1007/8904_2017 41.

9. Schollen E, Kjaergaard S, Legius E, Schwartz M, Matthijs G. Lack of hardyWeinberg equilibrium for the most prevalent PMM2 mutation in CDG-la (congenital disorders of glycosylation type la). Eur J Hum Genet. 2000;8(5): 367-71. https://doi.org/10.1038/sj.ejhg.5200470.

10. Belaya K, Rodriguez Cruz PM, Liu WW, Maxwell S, McGowan S, Farrugia ME, et al. Mutations in GMPPB cause congenital myasthenic syndrome and bridge myasthenic disorders with dystroglycanopathies. Brain. 2015;138(Pt 9):2493-504. https://doi.org/10.1093/brain/awv185

11. Rodriguez Cruz PM, Belaya K, Basiri K, Sedghi M, Farrugia ME, Holton JL, et al. Clinical features of the myasthenic syndrome arising from mutations in GMPPB. J Neurol Neurosurg Psychiatry. 2016;87(8):802-9. https://doi.org/ 10.1136/jnnp-2016-313163.

12. Luo S, Cai S, Maxwell S, Yue D, Zhu W, Qiao K, et al. Novel mutations in the C-terminal region of GMPPB causing limb-girdle muscular dystrophy overlapping with congenital myasthenic syndrome. Neuromuscul Disord. 2017;27(6):557-64. https://doi.org/10.1016/j.nmd.2017.03.004.

13. Carss KJ, Stevens E, Foley AR, Cirak S, Riemersma M, Torelli S, et al. Mutations in GDP-mannose pyrophosphorylase B cause congenital and limb-girdle muscular dystrophies associated with hypoglycosylation of alpha-dystroglycan. Am J Hum Genet. 2013;93(1):29-41. https://doi.org/10. 1016/j.ajhg.2013.05.009.

14. Cabrera-Serrano M, Ghaoui R, Ravenscroft G, Johnsen RD, Davis MR, Corbett A, et al. Expanding the phenotype of GMPPB mutations. Brain. 2015;138(Pt 4):836-44. https://doi.org/10.1093/brain/awv013.

15. Astrea G, Romano A, Angelini C, Antozzi CG, Barresi R, Battini R, et al. Broad phenotypic spectrum and genotype-phenotype correlations in GMPPBrelated dystroglycanopathies: an Italian cross-sectional study. Orphanet J Rare Dis. 2018;13(1):170. https://doi.org/10.1186/s13023-018-0863-x.

16. Jensen BS, Willer T, Saade DN, Cox MO, Mozaffar T, Scavina M, et al. GMPPBassociated Dystroglycanopathy: emerging common variants with phenotype correlation. Hum Mutat. 2015;36(12):1159-63. https://doi.org/10. 1002/humu.22898.

17. Nigro V, Savarese M. Genetic basis of limb-girdle muscular dystrophies: the 2014 update. Acta Myol. 2014;33(1):1-12.

18. Tian WT, Zhou HY, Zhan FX, Zhu ZY, Yang J, Chen SD, et al. Lysosomal degradation of GMPPB is associated with limb-girdle muscular dystrophy type 2T. Ann Clin Transl Neurol. 2019;6(6):1062-71. https:// doi.org/10.1002/acn3.787.

19. Mao W, Daligaux P, Lazar N, Ha-Duong T, Cave C, van Tilbeurgh H, et al. Biochemical analysis of leishmanial and human GDP-mannose Pyrophosphorylases and selection of inhibitors as new leads. Sci Rep. 2017; 7(1):751. https://doi.org/10.1038/s41598-017-00848-8. 
20. Luo N, Li H, Xiang B, Qiao L, He J, Ji Y, et al. Syndecan-4 modulates the proliferation of neural cells and the formation of $\mathrm{CaP}$ axons during zebrafish embryonic neurogenesis. Sci Rep. 2016;6:25300. https://doi.org/10.1038/ srep25300.

21. Arkhipova V, Wendik B, Devos N, Ek O, Peers B, Meyer D.

Characterization and regulation of the $\mathrm{hb} / \mathrm{mn} \times 1$ beta-cell progenitor specific enhancer in zebrafish. Dev Biol. 2012;365(1):290-302. https://doi. org/10.1016/j.ydbio.2012.03.001.

22. Raphael AR, Couthouis J, Sakamuri S, Siskind C, Vogel H, Day JW, et al. Congenital muscular dystrophy and generalized epilepsy caused by GMPPB mutations. Brain Res. 2014;1575:66-71. https://doi.org/10.1016/j.brainres. 2014.04.028.

23. Kimple ME, Brill AL, Pasker RL. Overview of affinity tags for protein purification. Curr Protoc Protein Sci. 2013;73:9 1-9 23. https://doi.org/10. 1002/0471140864.ps0909s73.

24. Thiel C, Lubke T, Matthijs G, von Figura K, Korner C. Targeted disruption of the mouse phosphomannomutase 2 gene causes early embryonic lethality. Mol Cell Biol. 2006;26(15):5615-20. https://doi.org/10.1128/MCB.02391-05.

25. Schneider A, Thiel C, Rindermann J, DeRossi C, Popovici D, Hoffmann GF, et al. Successful prenatal mannose treatment for congenital disorder of glycosylation-la in mice. Nat Med. 2011;18(1):71-3. https:// doi.org/10.1038/nm.2548.

26. Chan B, Clasquin M, Smolen GA, Histen G, Powe J, Chen Y, et al. A mouse model of a human congenital disorder of glycosylation caused by loss of PMM2. Hum Mol Genet. 2016;25(11):2182-93. https://doi.org/ 10.1093/hmg/ddw085.

27. Ravenscroft G, Thompson EM, Todd EJ, Yau KS, Kresoje N, Sivadorai P, et al. Whole exome sequencing in foetal akinesia expands the genotype-phenotype spectrum of GBE1 glycogen storage disease mutations. Neuromuscul Disord. 2013;23(2):165-9. https://doi.org/10. 1016/j.nmd.2012.11.005.

28. Wang $\mathrm{K}$, Li M, Hakonarson H. ANNOVAR: functional annotation of genetic variants from high-throughput sequencing data. Nucleic Acids Res. 2010; 38(16):e164. https://doi.org/10.1093/nar/gkq603.

29. Harrow J, Denoeud F, Frankish A, Reymond A, Chen CK, Chrast J, et al. GENCODE: producing a reference annotation for ENCODE. Genome Biol. 2006;7(Suppl 1):S4 1-9. https://doi.org/10.1186/gb-2006-7-s1-s4.

30. Qin J, Liu Q, Liu Z, Pan YZ, Sifuentes-Dominguez L, Stepien KP, et al. Structural and mechanistic insights into secretagogin-mediated exocytosis. Proc Natl Acad Sci U S A. 2020;117(12):6559-70. https://doi.org/10.1073/ pnas.1919698117.

31. Huang W, Liu Z, Yang F, Zhou H, Yong X, Yang X, et al. Structural and functional studies of TBC1D23 C-terminal domain provide a link between endosomal trafficking and PCH. Proc Natl Acad Sci U S A. 2019;116(45): 22598-608. https://doi.org/10.1073/pnas.1909316116.

\section{Publisher's Note}

Springer Nature remains neutral with regard to jurisdictional claims in published maps and institutional affiliations. 